\title{
EGU2020-20591
}

https://doi.org/10.5194/egusphere-egu2020-20591

EGU General Assembly 2020

(c) Author(s) 2020. This work is distributed under

the Creative Commons Attribution 4.0 License.

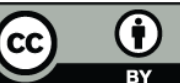

\section{The Italian TREETALKER NETWORK (ITT-Net): continuous large scale monitoring of tree functional traits and vulnerabilities to climate change}

Simona Castaldi ${ }^{2}$, Serena Antonucci ${ }^{6}$, Shahla Asgharina ${ }^{1}$, Giovanna Battipaglia ${ }^{2}$, Luca Belelli Marchesini $i^{3}$, Mauro Cavagna ${ }^{3}$, Isaac Chini ${ }^{3}$, Claudia Cocozza ${ }^{5}$, Damiano Gianelle ${ }^{3}$, Tommaso La Mantia ${ }^{7}$, Antonio Motisi ${ }^{7}$, Francesco Niccoli ${ }^{2}$, Arturo Pacheco Solana ${ }^{2}$, Giovanna Sala ${ }^{7}$, Giovanni Santopuoli ${ }^{6}$, Giustino Tonon ${ }^{4}$, Roberto Tognetti ${ }^{6}$, Roberto Zampedri ${ }^{3}$, Ilaria Zorzi ${ }^{5}$, and Riccardo Valentini ${ }^{1}$

${ }^{1}$ University of Tuscia, Viterbo, Italy

2University of Campania " Luigi Vanvitelli", Caserta, Italy

${ }^{3}$ Edmund Mach Foundation, Trento, Italy

${ }^{4}$ Free University of Bozen, Bozen, Italy

${ }^{5}$ University of Florence, Florence, Italy

${ }^{6}$ University of Molise, Campobasso, Italy

${ }^{7}$ University of Palermo, Palermo, Italy

The Italian TREETALKER NETWORK (ITT-Net) aims to respond to one of the grand societal challenges: the impact of climate changes on forests ecosystem services and forest dieback. The comprehension of the link between these phenomena requires to complement the most classical approaches with a new monitoring paradigm based on large scale, single tree, high frequency and long-term monitoring tree physiology, which, at present, is limited by the still elevated costs of multi-sensor devices, their energy demand and maintenance not always suitable for monitoring in remote areas. The ITT-Net network will be a unique and unprecedented worldwide example of real time, large scale, high frequency and long-term monitoring of tree physiological parameters. By spring 2020, as part of a national funded project (PRIN) the network will have set 37 sites from the north-east Alps to Sicily where a new low cost, multisensor technology "the TreeTalker ${ }^{\circledR}$ " equipped to measure tree radial growth, sap flow, transmitted light spectral components related to foliage dieback and physiology and plant stability (developed by Nature 4.0), will monitor over 600 individual trees. A radio LoRa protocol for data transmission and access to cloud services will allow to transmit in real time high frequency data on the WEB cloud with a unique IoT identifier to a common database where big data analysis will be performed to explore the causal dependency of climate events and environmental disturbances with tree functionality and resilience.

With this new network, we aim to create a new knowledge, introducing a massive data observation and analysis, about the frequency, intensity and dynamical patterns of climate anomalies perturbation on plant physiological response dynamics in order to: 1) characterize the space of "normal or safe tree operation mode" during average climatic conditions; 2 ) identify the non-linear 
tree responses beyond the safe operation mode, induced by extreme events, and the tipping points; 3 ) test the possibility to use a high frequency continuous monitoring system to identify early warning signals of tree stress which might allow to follow tree dynamics under climate change in real time at a resolution and accuracy that cannot always be provided through forest inventories or remote sensing technologies.

To have an overview of the ITT Network you can visit www.globaltreetalker.org 